\title{
Finding a Dense Subgraph with Sparse Cut
}

\author{
Atsushi Miyauchi \\ RIKEN AIP \\ Tokyo, Japan \\ atsushi.miyauchi.hv@riken.jp
}

\author{
Naonori Kakimura \\ Keio University \\ Kanagawa, Japan \\ kakimura@math.keio.ac.jp
}

\begin{abstract}
Community detection is one of the fundamental tasks in graph mining, which has many real-world applications in diverse domains. In this study, we propose an optimization model for finding a community that is densely connected internally but sparsely connected to the rest of the graph. The model extends the densest subgraph problem, in which we maximize the density while minimizing the average cut size. We first show that our proposed model can be solved efficiently. Then we design two polynomial-time exact algorithms based on linear programming and a maximum flow algorithm, respectively. Moreover, to deal with larger-sized graphs in practice, we present a scalable greedy algorithm that runs in almost linear time with theoretical performance guarantee of the output. In addition, as our model is closely related to a quality function called the modularity density, we show that our algorithms can also be used to find global community structure in a graph. With thorough experiments using well-known real-world graphs, we demonstrate that our algorithms are highly effective in finding a suitable community in a graph. For example, for web-Google, our algorithm finds a solution with more than $99.1 \%$ density and less than $3.1 \%$ cut size, compared with a solution obtained by a baseline algorithm for the densest subgraph problem.
\end{abstract}

\section{CCS CONCEPTS}

- Mathematics of computing $\rightarrow$ Graph algorithms; Approximation algorithms; Discrete optimization;

\section{KEYWORDS}

Graphs; community detection; densest subgraph; sparsest cut; modularity density; exact algorithms; approximation algorithms

\section{ACM Reference Format:}

Atsushi Miyauchi and Naonori Kakimura. 2018. Finding a Dense Subgraph with Sparse Cut. In The 27th ACM International Conference on Information and Knowledge Management (CIKM '18), October 22-26, 2018, Torino, Italy. ACM, New York, NY, USA, 10 pages. https://doi.org/10.1145/3269206.3271720

Permission to make digital or hard copies of all or part of this work for personal or classroom use is granted without fee provided that copies are not made or distributed for profit or commercial advantage and that copies bear this notice and the full citation on the first page. Copyrights for components of this work owned by others than ACM must be honored. Abstracting with credit is permitted. To copy otherwise, or republish, to post on servers or to redistribute to lists, requires prior specific permission and/or a fee. Request permissions from permissions@acm.org.

CIKM '18, October 22-26, 2018, Torino, Italy

(C) 2018 Association for Computing Machinery.

ACM ISBN 978-1-4503-6014-2/18/10 ..\$15.00

https://doi.org/10.1145/3269206.3271720

\section{INTRODUCTION}

Community detection is one of the fundamental tasks in graph mining, which has many real-world applications in diverse domains $[16,23]$. Intuitively speaking, a community in a graph is a subset of vertices densely connected internally but sparsely connected to the rest of the graph. For example, consider Web graphs, where vertices represent web pages and edges represent hyperlinks among them. Communities in Web graphs are very likely to correspond to the sets of pages dealing with similar topics [13] or spam link farms [22]. As another example, consider protein-protein interaction graphs, where vertices represent proteins in a cell and edges represent chemical interactions among them. Communities in such graphs correspond to the sets of proteins having similar functions [22].

To date, a large number of community detection methods have been developed in the field of computer science and statistical physics $[16,17]$. The most common way to detect a community is to utilize theory of optimization. In this approach, we first introduce a set function called a quality function on vertices, which hopefully captures the intuition of community structure of a graph. Specifically, a quality function returns some value representing how community-like a given subset of vertices is. With the quality function, we define an optimization model (i.e., optimization problem), and solve the model exactly or approximately to obtain a desired community in a graph. Chakraborty et al. [8] have recently provided an informative survey on quality functions for community detection.

One of the most popular quality function is the density, which is introduced based on the fact that a vertex in a community is closely connected to each other. Let us formally define the density function. Let $G=(V, E, w)$ be a simple undirected graph with edge weight $w: E \rightarrow \mathbb{R}_{>0}$, where $\mathbb{R}_{>0}$ is the set of positive real numbers. We denote by $G[S]$ the subgraph induced by $S \subseteq V$, i.e., $G[S]=(S, E(S))$, where $E(S)=\{\{u, v\} \in E \mid u, v \in S\}$. Then the density of a vertex subset $S \subseteq V$ is defined as $w_{\text {in }}[S] /|S|$, where $w_{\text {in }}[S]=\sum_{e \in E(S)} w_{e}$ is the sum of edge weights in $G[S]$. In other words, the density of $S \subseteq V$ is the half of the average (weighted) degree of $G[S]$. The weighted densest subgraph problem is to find a vertex subset $S \subseteq V$ that maximizes the density.

The densest subgraph problem has recently garnered much attention in practice due to its solvability. Indeed, the problem can be solved exactly in polynomial time by Goldberg's flow-based algorithm [24] and Charikar's LP-based algorithm [9]. Furthermore, Charikar [9] proved that a simple greedy algorithm, which follows the greedy peeling strategy by Asahiro et al. [4], admits $1 / 2$-approximation. The greedy algorithm can be implemented to run in $O(m+n \log n)$ time for weighted graphs and $O(m+n)$ time for unweighted graphs (i.e., $w_{e}=1$ for every $e \in E$ ), where $n=|V|$ and $m=|E|$. 


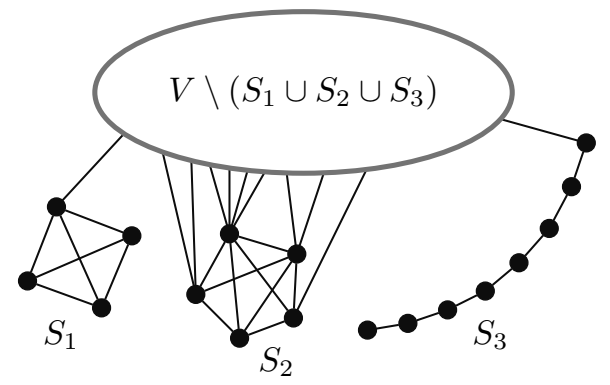

Figure 1: An example graph.

It is, however, known that the density function often fails to find a community. For example, consider a graph in Figure 1. Among $S_{1}, S_{2}$, and $S_{3}$, clearly $S_{2}$ has the largest density. However, $S_{1}$ is more community-like than $S_{2}$ because $S_{1}$ is much more sparsely connected to the rest of the graph. Thus, using the density is not necessarily enough to find a desired community.

On the other hand, as a community is sparsely connected to the rest of the graph, the cut function is often used for community detection. Let $\operatorname{cut}(S)$ be the set of cut edges of $S \subseteq V$, i.e., $\operatorname{cut}(S)=$ $\{\{u, v\} \in E \mid u \in S, v \in V \backslash S\}$. We denote by $w_{\text {cut }}[S]$ the sum of edge weights in $\operatorname{cut}(S)$, that is, $w_{\text {cut }}[S]=\sum_{e \in \operatorname{cut}(S)} w_{e}$, which we call the cut size of $S$.

A non-trivial community can be found by minimizing the cut size while keeping the vertex subset size large. This motivated us to introduce several quality functions such as the normalized cut, the ratio cut, the average cut, and the conductance. In particular, the average cut and the conductance have often been used in the analysis of community structure. The average cut of a vertex subset $S \subseteq V$ is defined as $w_{\text {cut }}[S] /|S|$. The problem of minimizing the average cut subject to $|S| \leq n / 2$ is called the sparsest cut problem, which is known to be NP-hard [21]. The best known approximation algorithm admits $O(\sqrt{\log n})$-approximation [3]. On the other hand, the conductance of a vertex subset $S \subseteq V$ is defined as $\phi(S)=$

$\frac{w_{\text {cut }}[S]}{\min \{\operatorname{vol}(S), \operatorname{vol}(V \backslash S)\}}$, where $\operatorname{vol}(S)$ is the sum of weighted degrees of the vertices in $S$. Conductance minimization is also known to be NP-hard even on unweighted graphs [44]. Leskovec et al. [32] proposed the network community profile plot (NCP plot) with the conductance, which was effectively used by Leskovec et al. [33] to compare a variety of community detection algorithms.

As both the average cut and the conductance focus only on the boundary of a subset and ignore its internal structure, it is impossible to find a dense subgraph by these methods. We remark that Leskovec et al. [33] states that the conductance considers the inside of subsets because it prefers $S \subseteq V$ with large $\operatorname{vol}(S)$; however, a large value of $\operatorname{vol}(S)$ does not necessarily guarantee the large density. For example, among $S_{1}, S_{2}$, and $S_{3}$ in Figure $1, S_{3}$ has the smallest conductance, while we would intuitively like to find $S_{1}$.

\subsection{Our Contribution}

The main purpose of this paper is to construct an optimization model for community detection that takes into account the density of the inside of a community as well as the sparsity of the boundary. We propose a new quality function combining the density function and the cut function as follows: For an edge-weighted undirected graph $G=(V, E, w)$ and a parameter $\alpha \in[0, \infty)$, define

$$
f_{\alpha}(S)=\frac{w_{\text {in }}[S]-\alpha \cdot w_{\text {cut }}[S]}{|S|} .
$$

Then we aim to find a vertex subset $S \subseteq V$ that maximizes the function $f_{\alpha}(S)$. As can be seen, we would like to maximize the density $w_{\text {in }}[S] /|S|$ while minimizing the average cut $w_{\text {cut }}[S] /|S|$. A parameter $\alpha \in[0, \infty)$ enables us to adjust the significance of the cut size term.

Problem 1 (Dense subgraph with sparse cut). Given an edgeweighted undirected graph $G=(V, E, w)$, we are asked to find $S \subseteq V$ that maximizes $f_{\alpha}(S)$.

It should be noted that a quality function similar to $f_{\alpha}$ is used in the context of global community detection (in which we aim to divide a graph into disjoint communities). Let $C=\left\{C_{1}, \ldots, C_{\ell}\right\}$ be a partition of $V$. That is, $C$ satisfies $\bigcup_{i=1}^{\ell} C_{i}=V, C_{i} \cap C_{j}=\emptyset$ for any $i \neq j$, and $C_{i} \neq \emptyset$ for any $i$. The modularity density, introduced by Li et al. [34, 35], is defined as

$$
D(C)=2 \sum_{C \in C} f_{1 / 2}(C)=\sum_{C \in C} \frac{2 \cdot w_{\text {in }}[C]-w_{\text {cut }}[C]}{|C|} .
$$

The problem of maximizing the modularity density has been studied to find global community structure $[11,12,42,43]$. Our model can be seen as a local community detection variant of the modularity density maximization.

Our contributions are summarized as follows, where the details are described below.

(i) We present two polynomial-time exact algorithms for Problem 1: an LP-based algorithm and a flow-based algorithm;

(ii) We design an almost-linear-time approximation algorithm with theoretical performance guarantee for Problem 1;

(iii) We show that our exact algorithms for Problem 1 can be used to maximize the modularity density $D(C)$ for global community detection;

(iv) We conduct thorough computational experiments to verify the validity of our optimization model and to evaluate the performance of our algorithms.

(i) We first observe that the numerator of $f_{\alpha}$ is supermodular (see Section 2 for the definition). Therefore, using a standard technique in fractional programming, we can reduce Problem 1 to a sequence of submodular function minimizations. This implies that Problem 1 is polynomially solvable. However, it is computationally expensive; even if we use the state-of-the-art submodular function minimization algorithm [30], it spends $O\left(n^{3}(m+n) \log ^{3} n+n^{4}\right.$ polylog $\left.(n)\right)$ time. To improve the running time, we design two more efficient exact algorithms by extending algorithms for the densest subgraph problem $[9,24]$. The first one is an LP-based algorithm. We design an LP problem by combining formulations of the densest subgraph problem and the minimum cut problem, which has $O(m+n)$ variables and $O(m+n)$ constraints. We then show that an optimal solution to the LP delivers an optimal solution to Problem 1 by appropriate rounding. This algorithm is simple to implement if we use a mathematical programming solver such as Gurobi Optimizer 
Table 1: A brief description of experimental results.

\begin{tabular}{|c|c|c|c|c|c|c|c|c|c|c|}
\hline \multirow[b]{2}{*}{ Graph } & \multirow[b]{2}{*}{$n$} & \multirow[b]{2}{*}{$m$} & \multicolumn{4}{|c|}{ Densest subgraph problem } & \multicolumn{4}{|c|}{ Our problem $(\alpha=0.2)$} \\
\hline & & & $|S|$ & $\frac{2 \cdot w_{\text {in }}[S]}{|S|}$ & $w_{\text {cut }}[S]$ & $\frac{w_{\text {cut }}[S \mid}{|S|}$ & $|S|$ & $\frac{2 \cdot w_{\text {in }}[S]}{|S|}$ & $w_{\text {cut }}[S]$ & $\frac{w_{\text {cut }}[S]}{|S|}$ \\
\hline web-Stanford & 255,265 & $1,941,926$ & 547 & 118.37 & 30,748 & 56.21 & 830 & 113.57 & 18,757 & 22.60 \\
\hline web-NotreDame & 325,729 & $1,090,108$ & 1,367 & 157.32 & 37,415 & 27.37 & 451 & 151.34 & 1,805 & 4.00 \\
\hline web-Berkstan & 654,782 & $6,581,871$ & 392 & 206.81 & 236,523 & 603.38 & 4,592 & 167.16 & 46,922 & 10.22 \\
\hline web-Google & 855,802 & $4,291,352$ & 240 & 54.36 & 10,524 & 43.85 & 61 & 53.90 & 316 & 5.18 \\
\hline
\end{tabular}

or IBM ILOG CPLEX. The second one is a flow-based algorithm. We prove that Problem 1 can be solved by a sequence of minimum cut computations. Both of our algorithms run in polynomial time. The first one runs fast in practice due to the power of state-of-the-art mathematical programming solvers. The second one runs in $O\left(n^{3}\right)$ time for unweighted graphs.

(ii) Although our exact algorithms are more efficient than the algorithm based on submodular function minimization, they are not sufficiently scalable in practice; indeed, they are not applicable to graphs with millions of edges. To design a more scalable algorithm, we aim to find an approximate solution in almost linear time based on a greedy strategy. Our algorithm iteratively removes a vertex to obtain a sequence of vertex subsets from the entire set to the empty set, and then returns the best subset among them. This kind of greedy algorithm is called a peeling algorithm. We remark that peeling algorithms have been proposed for the densest subgraph problem and its variations $[1,4,28,45]$ as well as other related problems $[39,46]$. The difference between existing algorithms and ours is the criterion for choosing a vertex to be removed in each iteration; all existing algorithms just remove a vertex with minimum degree, while our proposed algorithm takes into account not only the degree of a vertex but also the cut edges connected to a vertex. As will be demonstrated in our experiments (in Section 6), the difference is essential. In fact, our algorithm often obtains a dense subgraph with particularly small cut that does not appear in the sequence of subsets produced by the standard greedy peeling strategy. It should be noted that our algorithm has theoretical performance guarantee of the output and runs in $O(m+n \log n)$ time.

(iii) The $k$-modularity density maximization problem is that, given an edge-weighted undirected graph $G=(V, E, w)$ and a positive integer $k$, we are asked to find a partition $C$ of $V$ with size $|C| \leq k$ that maximizes the modularity density $D(C)$. With the aid of our exact algorithms in (i), we propose a polynomial-time approximation algorithm for the $k$-modularity density maximization problem. Specifically, our algorithm iteratively extracts $S \subseteq V$ that maximizes our quality function $f_{1 / 2}(S)$ as long as the number of clusters is less than $k$ and the modularity density increases. We prove that our algorithm admits $\frac{2}{3 k}$-approximation. It should be noted that this is the first polynomial-time approximation algorithm for an optimization problem related to modularity density maximization.

(iv) In a series of experimental assessments, we verify the validity of our optimization model and evaluate the performance of our proposed algorithms using a variety of well-known real-world graphs. We demonstrate that our proposed algorithms are highly effective in obtaining a dense subgraph with sparse cut. Table 1 provides a brief description of experimental results for famous Web graphs with unweighted edges. We compare solutions obtained by our proposed greedy peeling algorithm for Problem 1 with solutions obtained by a standard approximation algorithm for the densest subgraph problem $[4,9]$. As can be seen, our algorithm finds a solution with as almost the same density as the baseline solution, that has a much smaller cut size. In particular, for web-NotreDame and web-Google, our algorithm finds $S \subseteq V$ with more than $96.1 \%$ density and less than $4.8 \%$ cut size and $S \subseteq V$ with more than $99.1 \%$ density and less than $3.1 \%$ cut size, respectively.

\subsection{Related Work}

In addition to its original form, the densest subgraph problem has a large number of problem variations and generalizations. The most well-studied ones are the size-restricted problems such as the densest $k$-subgraph problem, i.e., the problem of finding a set of $k$ vertices that maximizes the density. The problem includes the $k$-clique problem and thus is much harder than the densest subgraph problem. For size-restricted densest subgraph problems, various approximation algorithms have been developed [1, 6, 15, 29]. There are some studies that make modifications on the numerator $w_{\text {in }}[S]$ of the density [37, 45] and the denominator $|S|$ of the density [28] for some specific purposes. The densest subgraph problem is generalized to those on directed graphs [9], hypergraphs [25, 38], multilayer graphs [20], and uncertain graphs [47]. The problem is also studied in dynamic settings $[14,25,40]$ and streaming settings [2, 5, 7, 36].

As explained above, our quality function can be seen as a local community detection variant of the modularity density [34, 35]. In a similar manner, Miyauchi and Kawase [39] recently introduced a local community detection variant of the modularity [41], which they call the communitude. They provided a greedy peeling algorithm together with a simple local search for the communitude maximization problem. We remark that their algorithm still follows the standard greedy peeling strategy by Asahiro et al. [4], which is different from ours.

The modularity density maximization problem has been studied in the context of global community detection [11, 12, 42, 43]. It should be noted that, in the derivation process of the modularity density, our quality function with $\alpha=1 / 2$ was considered by Li et al. [34, 35]. However, their interest was in global community detection, and our proposed model is the first to investigate local community structure using the function. Costa et al. [12] designed a hierarchical divisive heuristic for the modularity density maximization problem that iteratively solves the $k$-modularity density maximization problem with $k=2$. 
Huang and Kahng [26] studied the $k$-way maximum sum of densities ( $k$-MSD). In this problem, given an edge-weighted undirected graph $G=(V, E, w)$, we are asked to find a partition $C$ of $V$ with size $|C| \leq k$ that maximizes the sum of densities $\sum_{C \in C} \frac{w_{\text {in }}[C]}{|C|}$. This problem can be viewed as the $k$-modularity density maximization problem without the cut size term. They proposed a $1 / k-$ approximation algorithm for the $k$-MSD. On the other hand, the problem was shown to be NP-hard when the size of a partition is exactly equal to $k$.

\section{DENSE SUBGRAPH WITH SPARSE CUT}

Let $G=(V, E, w)$ be an edge-weighted undirected graph. Recall that Problem 1 is to find $S \subseteq V$ that maximizes the following quality function:

$$
f_{\alpha}(S)=\frac{w_{\text {in }}[S]-\alpha \cdot w_{\text {cut }}[S]}{|S|} \quad(\alpha \in[0, \infty)) .
$$

Throughout the paper, we assume that $E \neq \emptyset$. Thus, letting $S^{*} \subseteq V$ be an optimal solution to the problem, we have $\left|S^{*}\right| \geq 2$. For $S \subseteq V$ and $v \in S$, let $d_{S}(v)$ be the weighted degree of $v$ on $G[S]$, i.e., $d_{S}(v)=\sum_{e=\{u, v\} \in E(S)} w_{e}$. When $S=V$, we simply denote $d_{V}(v)$ by $d(v)$. Let $d_{\max }=\max _{v \in V} d(v)$ and $w_{\min }=\min _{e \in E} w_{e}$.

Depending on the value of parameter $\alpha \in[0, \infty)$, the problem can vary as follows. If $\alpha=0$, then the problem is identical with the densest subgraph problem. On the other hand, the following proposition states that a sufficiently large $\alpha$ makes the problem trivial.

Proposition 1. If $\alpha \geq \frac{n \cdot d_{\max }-2 \cdot w_{\text {in }}[V]}{2 \cdot w_{\min }}$ holds, then a connected component with the largest density in $G$ is optimal to Problem 1.

Proof. Let us choose an arbitrary $S^{\prime} \subseteq V$ such that $\operatorname{cut}\left(S^{\prime}\right) \neq \emptyset$. Then we have

$$
\begin{aligned}
f_{\alpha}\left(S^{\prime}\right) & =\frac{w_{\text {in }}\left[S^{\prime}\right]-\alpha \cdot w_{\text {cut }}\left[S^{\prime}\right]}{\left|S^{\prime}\right|} \leq \frac{w_{\text {in }}\left[S^{\prime}\right]}{\left|S^{\prime}\right|}-\alpha \cdot \frac{w_{\text {min }}}{\left|S^{\prime}\right|} \\
& <\frac{d_{\text {max }}}{2}-\alpha \cdot \frac{w_{\min }}{n} \leq \frac{d_{\text {max }}}{2}-\frac{n \cdot d_{\text {max }}-2 \cdot w_{\text {in }}[V]}{2 n} \\
& =\frac{w_{\text {in }}[V]}{n}=f_{\alpha}(V),
\end{aligned}
$$

where the second inequality follows from the facts that $\frac{w_{\text {in }}[S]}{|S|} \leq$ $d_{\max } / 2$ for any $S \subseteq V, w_{\min }>0$, and $\left|S^{\prime}\right|<n$, and the third inequality follows from the choice of $\alpha$. Therefore, we see that $S^{\prime}$ is not optimal. Noticing that any solution consisting of more than one connected components contains at least one component with no smaller objective value, we have the theorem.

Let $g: 2^{V} \rightarrow \mathbb{R}$ be a set function defined on a finite set $V$. A set function $g$ is said to be submodular if it satisfies $g(X)+g(Y) \geq$ $g(X \cup Y)+g(X \cap Y)$ for all $X, Y \subseteq V$. A set function $g$ is supermodular if $-g$ is submodular, and modular if $g$ is submodular and supermodular. It is well known that submodular functions can be minimized in polynomial time. See e.g., $[19,27]$ for the details. For an edge-weighted graph $G=(V, E, w)$, it is known that $w_{\text {in }}$ is a supermodular function, while $w_{\text {cut }}$ is a submodular function (see Fujishige [19]).

Here we show that Problem 1 can be reduced to a sequence of submodular function minimizations. To this end, we consider

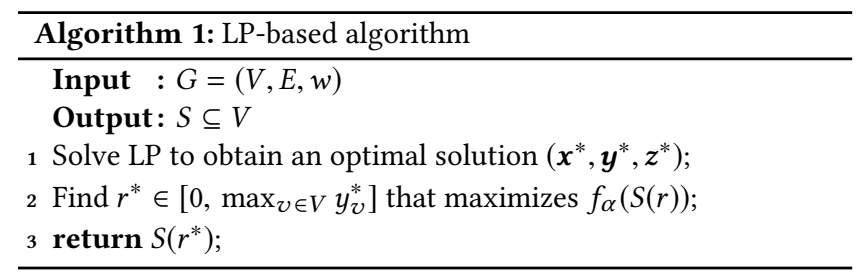

deciding whether $\max _{S \subseteq V} f_{\alpha}(S) \geq \beta$ holds for a given real parameter $\beta \geq 0$. By the definition of $f_{\alpha}$, it holds that $f_{\alpha}(S) \geq \beta$ if and only if $-w_{\text {in }}[S]+\alpha \cdot w_{\text {cut }}[S]+\beta \cdot|S| \leq 0$. We observe that the function $g(S)=-w_{\text {in }}[S]+\alpha \cdot w_{\text {cut }}[S]+\beta \cdot|S|$ is submodular because $-w_{\text {in }}[S]$ and $\alpha \cdot w_{\text {cut }}[S]$ are submodular and $\beta \cdot|S|$ is modular. Hence, we can decide whether there exists $S \subseteq V$ such that $g(S) \leq 0$ by minimizing the submodular function $g$. This can be done in $O\left(n^{3}(m+n) \log ^{2} n+n^{4}\right.$ polylog $\left.(n)\right)$ time using the state-ofthe-art submodular function minimization algorithm [30]. Thus, we can decide whether $\max _{S \subseteq V} f_{\alpha}(S) \geq \beta$ holds or not in the same time complexity. Since the optimal value is contained in the interval of $\left[f_{\alpha}(V) / n, d_{\max } / 2\right]$, we can find an optimal solution in polynomial time by the binary search over the interval. Note that, for unweighted graphs, the objective function $f_{\alpha}$ takes at most $O\left(m^{2} n\right)$ distinct values because both $w_{\text {in }}[S]$ and $w_{\text {cut }}[S]$ take at most $m+1$ distinct values from 0 to $m$ and $|S|$ takes at most $n$ distinct values from 1 to $n$. Thus, we can apply the binary search for the possible objective function values, and the number of iterations is $O\left(\log \left(m^{2} n\right)\right)=O(\log n)$. In this case, the total running time is $O\left(n^{3}(m+n) \log ^{3} n+n^{4}\right.$ polylog $\left.(n)\right)$.

\section{EXACT ALGORITHMS}

In this section, we propose two exact algorithms for Problem 1.

\subsection{LP-Based Algorithm}

In this subsection, we present our LP-based exact algorithm. We introduce a variable $y_{v}$ for each $v \in V$ and two variables $x_{e}$ and $z_{e}$ for each $e \in E$. We define the following LP problem:

$$
\begin{aligned}
& \text { LP: } \max . \sum_{e \in E} w_{e}\left(x_{e}-\alpha \cdot z_{e}\right) \\
& \text { s.t. } x_{e} \leq y_{u}, x_{e} \leq y_{v} \quad(\forall e=\{u, v\} \in E), \\
& y_{u}-y_{v} \leq z_{e}, y_{v}-y_{u} \leq z_{e} \quad(\forall e=\{u, v\} \in E), \\
& \sum_{v \in V} y_{v}=1 \text {, } \\
& x_{e}, y_{v}, z_{e} \geq 0
\end{aligned}
$$

Our algorithm first solves LP to obtain an optimal solution $\left(\boldsymbol{x}^{*}, \boldsymbol{y}^{*}, \boldsymbol{z}^{*}\right)$, and then computes $r^{*} \in\left[0, \max _{v \in V} y_{v}^{*}\right]$ that maximizes $f_{\alpha}(S(r))$, where $S(r)=\left\{v \in V \mid y_{v}^{*} \geq r\right\}$ for a real number $r \geq 0$. Note that to find $r^{*} \in\left[0, \max _{v \in V} y_{v}^{*}\right]$ that maximizes $f_{\alpha}(S(r))$, it suffices to check all distinct subsets $S(r)$ by simply setting $r=y_{v}^{*}$ for every $v \in V$. Finally, the algorithm returns $S\left(r^{*}\right)$. For reference, we describe the procedure in Algorithm 1. Clearly, the algorithm runs in polynomial time.

In what follows, we prove that Algorithm 1 obtains an optimal solution to Problem 1. Now we assume $\alpha>0$. If $\alpha=0$, our algorithm is identical to Charikar's LP-based exact algorithm for the densest 
subgraph problem [9]; hence, we are done. The following lemma provides a lower bound on the optimal value of LP.

Lemma 1. For any $S \subseteq V$, the optimal value of $L P$ is at least $f_{\alpha}(S)$.

Proof. Construct a solution $(\boldsymbol{x}, \boldsymbol{y}, \boldsymbol{z})$ of LP as follows: $x_{e}=\frac{1}{|S|}$ if $e \in E(S)$ and 0 otherwise, $y_{v}=\frac{1}{|S|}$ if $v \in S$ and 0 otherwise, and $z_{e}=\frac{1}{|S|}$ if $e \in \operatorname{cut}(S)$ and 0 otherwise. Then we can check that $(\boldsymbol{x}, \boldsymbol{y}, \boldsymbol{z})$ is feasible for LP. Indeed, the first constraints in LP are satisfied because for any $e=\{u, v\} \in E, e$ is contained in $E(S)$ if and only if both $u$ and $v$ are contained in $S$. The second constraints in LP are satisfied because for any $e=\{u, v\} \in E$, either $u$ or $v$ is contained in $S$ if and only if $e$ is contained in $\operatorname{cut}(S)$. The third constraint is satisfied because $\sum_{v \in V} y_{v}=\sum_{v \in S} y_{v}=1$. The objective value of $(\boldsymbol{x}, \boldsymbol{y}, \boldsymbol{z})$ is equal to

$$
\begin{aligned}
\sum_{e \in E} w_{e}\left(x_{e}-\alpha \cdot z_{e}\right) & =\frac{\sum_{e \in E(S)} w_{e}-\alpha \sum_{e \in \operatorname{cut}(S)} w_{e}}{|S|} \\
& =\frac{w_{\text {in }}[S]-\alpha \cdot w_{\text {cut }}[S]}{|S|}=f_{\alpha}(S) .
\end{aligned}
$$

Thus we have the lemma.

Using the above lemma, we prove the following key lemma.

Lemma 2. Let $S^{*} \subseteq V$ be an optimal solution to Problem 1. There exists $r^{*} \in\left[0, \max _{v \in V} y_{v}^{*}\right]$ that satisfies $f_{\alpha}\left(S\left(r^{*}\right)\right) \geq f_{\alpha}\left(S^{*}\right)$.

Proof. From the optimality of $\left(\boldsymbol{x}^{*}, \boldsymbol{y}^{*}, z^{*}\right)$, we have that $x_{e}^{*}=$ $\min \left\{y_{u}, y_{v}\right\}$ and $z_{e}^{*}=\left|y_{u}^{*}-y_{v}^{*}\right|$ for each $e=\{u, v\} \in E$. For simplicity, we denote $\max _{v \in V} y_{v}^{*}$ by $y_{\max }^{*}$.

For each $v \in V$, we define an indicator function $I_{v}:\left[0, y_{\max }^{*}\right] \rightarrow$ $\{0,1\}$ such that $I_{v}(r)=1$ if $r \leq y_{v}^{*}$ and 0 otherwise. In addition, for each $e=\{u, v\} \in E$, we define two indicator functions $J_{e}$ : $\left[0, y_{\max }^{*}\right] \rightarrow\{0,1\}$ and $K_{e}:\left[0, y_{\max }^{*}\right] \rightarrow\{0,1\}$ such that $J_{e}(r)=1$ if $r \leq \min \left\{y_{u}^{*}, y_{v}^{*}\right\}$ and 0 otherwise, and $K_{e}(r)=1$ if $y_{u}^{*}<r \leq y_{v}^{*}$ or $y_{v}^{*}<r \leq y_{u}^{*}$ and 0 otherwise. Using these functions, we have the following two equalities:

$$
\begin{aligned}
\int_{0}^{y_{\max }^{*}}|S(r)| d r & =\int_{0}^{y_{\max }^{*}}\left(\sum_{v \in V} I_{v}(r)\right) d r \\
& =\sum_{v \in V}\left(\int_{0}^{y_{\max }^{*}} I_{v}(r) d r\right)=\sum_{v \in V} y_{v}^{*}=1
\end{aligned}
$$

and

$$
\begin{aligned}
& \int_{0}^{y_{\max }^{*}}\left(w_{\text {in }}[S(r)]-\alpha \cdot w_{\text {cut }}[S(r)]\right) d r \\
& =\int_{0}^{y_{\max }^{*}}\left(\sum_{e \in E} w_{e}\left(J_{e}(r)-\alpha \cdot K_{e}(r)\right)\right) d r \\
& =\sum_{e \in E} w_{e}\left(\int_{0}^{y_{\max }^{*}}\left(J_{e}(r)-\alpha \cdot K_{e}(r)\right) d r\right) \\
& =\sum_{e=\{u, v\} \in E} w_{e}\left(\min \left\{y_{u}^{*}, y_{v}^{*}\right\}-\alpha \cdot\left|y_{u}^{*}-y_{v}^{*}\right|\right) \\
& =\sum_{e \in E} w_{e}\left(x_{e}^{*}-\alpha \cdot z_{e}^{*}\right) .
\end{aligned}
$$

Let $r^{*} \in\left[0, y_{\max }^{*}\right]$ be a real number that maximizes $f_{\alpha}(S(r))$. Then we have

$$
\begin{aligned}
f_{\alpha}\left(S^{*}\right) & \leq \sum_{e \in E} w_{e}\left(x_{e}^{*}-\alpha \cdot z_{e}^{*}\right) \\
& =\int_{0}^{y_{\max }^{*}}\left(w_{\text {in }}[S(r)]-\alpha \cdot w_{\text {cut }}[S(r)]\right) d r \\
& =\int_{0}^{y_{\max }^{*}}\left(\frac{w_{\text {in }}[S(r)]-\alpha \cdot w_{\text {cut }}[S(r)]}{|S(r)|} \cdot|S(r)|\right) d r \\
& \leq \int_{0}^{y_{\max }^{*}}\left(\frac{w_{\text {in }}\left[S\left(r^{*}\right)\right]-\alpha \cdot w_{\text {cut }}\left[S\left(r^{*}\right)\right]}{\left|S\left(r^{*}\right)\right|} \cdot|S(r)|\right) d r \\
& =\left(\frac{w_{\text {in }}\left[S\left(r^{*}\right)\right]-\alpha \cdot w_{\text {cut }}\left[S\left(r^{*}\right)\right]}{\left|S\left(r^{*}\right)\right|}\right) \int_{0}^{y_{\max }^{*}}|S(r)| d r \\
& =f_{\alpha}\left(S\left(r^{*}\right)\right),
\end{aligned}
$$

where the first inequality follows from Lemma 1, the first equality follows from equality (2), the second inequality follows from the definition of $r^{*}$, and the last equality follows from equality (1). Thus, we have the lemma.

The above lemma guarantees that our LP-based algorithm finds an optimal solution to Problem 1. We summarize our result in the following theorem.

Theorem 1. Our LP-based algorithm (Algorithm 1) finds an optimal solution to Problem 1 in polynomial time.

\subsection{Flow-Based Algorithm}

In this subsection, we present a flow-based exact algorithm. As mentioned in Section 2, to solve Problem 1, it suffices to decide whether $\max _{S \subseteq V} f_{\alpha}(S) \geq \beta$ holds for a real parameter $\beta \geq 0$. That is, we consider testing whether there exists $S \subseteq V$ such that $-w_{\text {in }}[S]+\alpha \cdot w_{\text {cut }}[S]+\beta \cdot|S| \leq 0$. In what follows, we show that such a set $S$ can be found by a flow-based algorithm (if exists).

From a given graph $G=(V, E, w)$, we construct an edge-weighted directed network $\left(U, A, w_{\beta}\right)$ as follows:

- $U=V \cup\{s, t\}$;

- $A=A_{1} \cup A_{2} \cup A_{3}$ such that $A_{1}=\{(s, v) \mid v \in V\}, A_{2}=$ $\{(u, v),(v, u) \mid\{u, v\} \in E\}$, and $A_{3}=\{(v, t) \mid v \in V\}$;

- $w_{\beta}: A \rightarrow \mathbb{R}_{\geq 0}$ such that

$$
w_{\beta}(e)= \begin{cases}\frac{d(v)}{2} & \text { if } e=(s, v) \in A_{1}, \\ \left(\frac{1}{2}+\alpha\right) \cdot w(\{u, v\}) & \text { if } e=(u, v) \in A_{2}, \\ \beta & \text { if } e=(v, t) \in A_{3} .\end{cases}
$$

Note that $w_{\beta}(e) \geq 0$ holds for every $e \in A$.

Recall that a bisection $(X, Y)$ of $U$ is called an $s-t$ cut of $\left(U, A, w_{\beta}\right)$ if $s \in X$ and $t \in Y$ hold. The cost of $s-t$ cut $(X, Y)$ is defined by $\sum_{e=(u, v) \in A: u \in X, v \in Y} w_{\beta}(u, v)$. The following lemma reveals the relationship between the cost of $s-t$ cut in the network and the value of $-w_{\text {in }}[S]+\alpha \cdot w_{\text {cut }}[S]+\beta \cdot|S|$.

Lemma 3. Let $(X, Y)$ be any $s-t$ cut of $\left(U, A, w_{\beta}\right)$ and $S=X \backslash\{s\}$. Then the cost of $(X, Y)$ is equal to

$$
w_{\text {in }}[V]-w_{\text {in }}[S]+\alpha \cdot w_{\text {cut }}[S]+\beta \cdot|S| .
$$




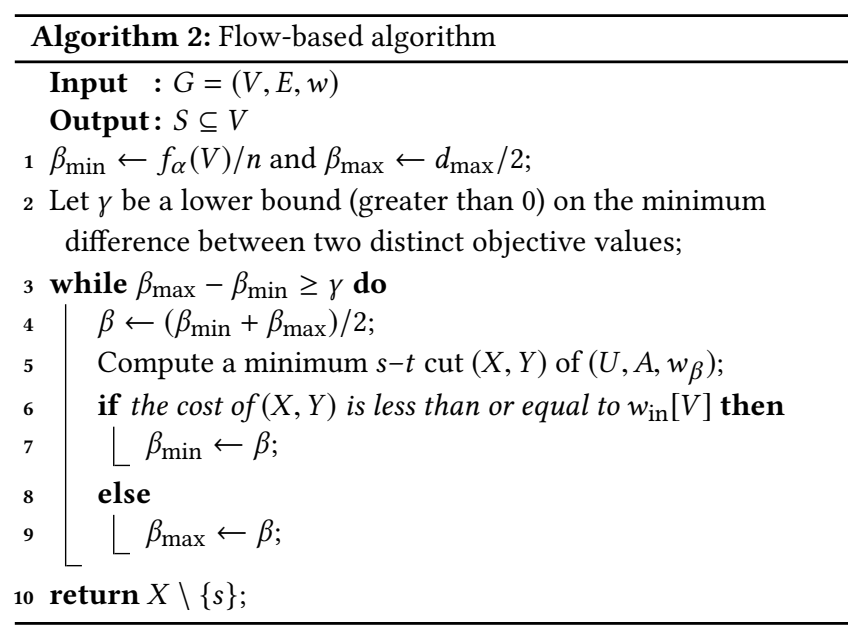

Proof. The set of edges from the vertices in $X$ to the vertices in $Y$ is given by

$$
\begin{gathered}
\left\{(s, v) \in A_{1} \mid v \in V \backslash S\right\} \cup\left\{(u, v) \in A_{2} \mid u \in S, v \in V \backslash S\right\} \\
\cup\left\{(v, t) \in A_{3} \mid v \in S\right\} .
\end{gathered}
$$

Therefore, the cost of $(X, Y)$ is equal to

$$
\begin{aligned}
& \frac{1}{2} \sum_{v \in V \backslash S} d(v)+\left(\frac{1}{2}+\alpha\right) \sum_{e \in \operatorname{cut}(S)} w_{e}+\beta \cdot|S| \\
& =w_{\text {in }}[V]-w_{\text {in }}[S]+\alpha \cdot w_{\text {cut }}[S]+\beta \cdot|S| .
\end{aligned}
$$

From this lemma, we see that the cost of a minimum $s-t$ cut is equal to

$$
w_{\text {in }}[V]+\min _{S \subseteq V}\left(-w_{\text {in }}[S]+\alpha \cdot w_{\text {cut }}[S]+\beta \cdot|S|\right) .
$$

Thus, we can decide whether $\max _{S \subseteq V} f_{\alpha}(S) \geq \beta$ by checking whether the cost of a minimum $s-t$ cut of $\left(U, A, w_{\beta}\right)$ is less than or equal to $w_{\text {in }}[V]$.

For reference, we describe the procedure together with the binary search in Algorithm 2. We set a parameter $\gamma$ to be a lower bound (greater than 0 ) on the minimum difference between two distinct objective values. We note that the parameter $\gamma$ can be represented using a polynomial on the input. In fact, letting $w_{e}$ be a rational number $p_{e} / q_{e}$ for every $e \in E$, where $p_{e}$ is an integer and $q_{e}$ is a positive integer, we see that the minimum difference between two distinct objective values is at least $1 /\left(n^{2} \prod_{e \in E} q_{e}\right)$ if $\alpha$ is integer and $\min \{\lceil\alpha\rceil-\alpha, \alpha-\lfloor\alpha\rfloor\} /\left(n^{2} \prod_{e \in E} q_{e}\right)$ otherwise. Clearly, the algorithm runs in polynomial time.

Note that, for unweighted graphs, we can apply the binary search for possible objective function values, and the number of iterations is $O(\log n)$, as mentioned in Section 2. Noticing that a minimum $s-t$ cut of an edge-weighted directed network with $N$ vertices can be computed in $O\left(N^{3} / \log N\right)$ time [10], we see that the total running time is $O\left(n^{3}\right)$ since $|U|=n+2$, which significantly improves the running time of the algorithm based on submodular function minimization in Section 2.

We summarize our result in the following theorem.

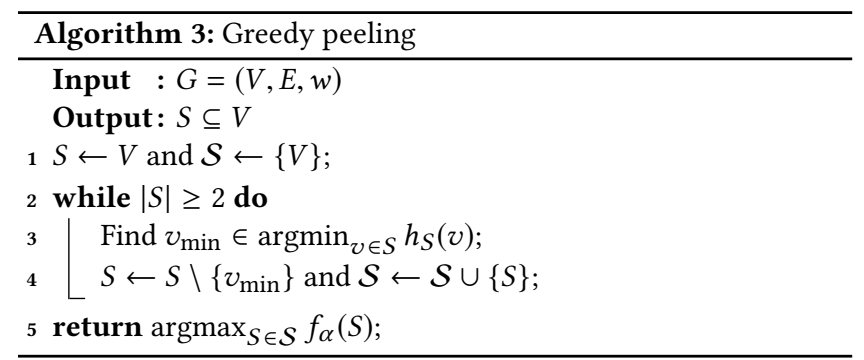

Theorem 2. Our flow-based algorithm (Algorithm 2) finds an optimal solution to Problem 1 in polynomial time. In particular, it runs in $O\left(n^{3}\right)$ time for unweighted graphs.

\section{APPROXIMATION ALGORITHM}

In this section, we present an almost-linear-time approximation algorithm. As described in Section 1, we employ a greedy peeling strategy taking into account not only the degree of a vertex but also the cut edges connected to a vertex.

Let $S \subseteq V$ be a vertex subset. Then, for each vertex $v \in S$, consider

$$
h_{S}(v)=\frac{1}{2} \cdot d_{S}(v)-\alpha \cdot d_{V \backslash(S \backslash\{v\})}(v) .
$$

The first term is the half of the degree of $v$ in $G[S]$, and the second term is the number of edges from $v$ to $V \backslash S$ multiplied by $-\alpha$.

Our algorithm starts from $S=V$. It iteratively removes a vertex $v$ from $S$ that minimizes $h_{S}(v)$. In the end, we obtain a sequence of subsets, and return the subset with maximum $f_{\alpha}(S)$ among them. A pseudo-code is described in Algorithm 3. Using the Fibonacci heap [18] to manage the remaining vertices over the iterations, we can implement the algorithm to run in $O(m+n \log n)$ time.

The following theorem provides the theoretical performance guarantee of our algorithm.

Theorem 3. Our greedy peeling algorithm (Algorithm 3) outputs $S \subseteq V$ that satisfies

$$
f_{\alpha}(S) \geq \frac{1}{2(1+\alpha)} \cdot f_{\alpha}\left(S^{*}\right)-\frac{\alpha(1+2 \alpha)}{2(1+\alpha)} \cdot d_{V \backslash\left(S^{*} \backslash\left\{v^{*}\right\}\right)}\left(v^{*}\right),
$$

where $S^{*} \subseteq V$ is an optimal solution to Problem 1, and $v^{*}$ is the vertex contained in $S^{*}$ that is removed first by Algorithm 3.

Proof. Choose an arbitrary vertex $v \in S^{*}$. From the optimality of $S^{*} \subseteq V$, it holds that

$$
\frac{w_{\text {in }}\left[S^{*}\right]-\alpha \cdot w_{\text {cut }}\left[S^{*}\right]}{\left|S^{*}\right|} \geq \frac{w_{\text {in }}\left[S^{*} \backslash\{v\}\right]-\alpha \cdot w_{\text {cut }}\left[S^{*} \backslash\{v\}\right]}{\left|S^{*}\right|-1} .
$$

By using the facts that $w_{\text {in }}\left[S^{*} \backslash\{v\}\right]=w_{\text {in }}[S]-d_{S^{*}}(v)$ and $w_{\text {cut }}\left[S^{*} \backslash\right.$ $\{v\}]=w_{\text {cut }}\left[S^{*}\right]+d_{S^{*}}(v)-d_{V \backslash\left(S^{*} \backslash\{v\}\right)}(v)$, the above inequality can be transformed into

$$
\begin{aligned}
(1+\alpha) \cdot d_{S^{*}}(v)-\alpha \cdot d_{V \backslash\left(S^{*} \backslash\{v\}\right)}(v) & \geq \frac{w_{\text {in }}\left[S^{*}\right]-\alpha \cdot w_{\text {cut }}\left[S^{*}\right]}{\left|S^{*}\right|} \\
& =f_{\alpha}\left(S^{*}\right) .
\end{aligned}
$$


Let $S^{\prime} \subseteq V$ be the last subset over the iterations that contains $v^{*}$. Then we have

$$
\begin{aligned}
f_{\alpha}\left(S^{\prime}\right)= & \frac{\sum_{v \in S^{\prime}} h_{S^{\prime}}(v)}{\left|S^{\prime}\right|} \geq h_{S^{\prime}}\left(v^{*}\right) \geq h_{S^{*}}\left(v^{*}\right) \\
= & \frac{1}{2(1+\alpha)} \cdot\left((1+\alpha) \cdot d_{S^{*}}\left(v^{*}\right)-\alpha \cdot d_{V \backslash\left(S^{*} \backslash\left\{v^{*}\right\}\right)}\left(v^{*}\right)\right) \\
& -\frac{\alpha(1+2 \alpha)}{2(1+\alpha)} \cdot d_{V \backslash\left(S^{*} \backslash\left\{v^{*}\right\}\right)}\left(v^{*}\right) \\
\geq & \frac{1}{2(1+\alpha)} \cdot f_{\alpha}\left(S^{*}\right)-\frac{\alpha(1+2 \alpha)}{2(1+\alpha)} \cdot d_{V \backslash\left(S^{*} \backslash\left\{v^{*}\right\}\right)}\left(v^{*}\right),
\end{aligned}
$$

where the first inequality follows from the greedy choice of $v^{*}$, the second inequality follows from $S^{\prime} \supseteq S^{*}$, and the last inequality follows from inequality (3). Noticing that the algorithm considers $S^{\prime}$ as a candidate subset of the output, we have the theorem.

The above approximation guarantee is effective because the parameter $\alpha$ is usually small. As will be demonstrated in our experiments, even if we aim to obtain a dense subgraph with particularly small cut, it suffices to set $\alpha$ to be a small value (e.g., 0.2). This makes the first term of the approximation bound large and the second term small.

\section{GLOBAL COMMUNITY DETECTION}

In this section, we extend our community detection algorithms to global community detection. Specifically, we utilize our exact algorithms for Problem 1 to design an approximation algorithm for the $k$-modularity density maximization problem, which is defined as follows:

Problem 2 ( $k$-modularity density maximization problem). Given an edge-weighted undirected graph $G=(V, E, w)$ and a positive integer $k$, we are asked to find a partition $C$ with size $|C| \leq k$ that maximizes the modularity density

$$
D(C)=2 \sum_{C \in C} f_{1 / 2}(C)=\sum_{C \in C} \frac{2 \cdot w_{\text {in }}[C]-w_{\text {cut }}[C]}{|C|} .
$$

We first consider the case where $k=2$. Our algorithm computes $S^{*} \in \operatorname{argmax}_{S \subseteq V} f_{1 / 2}(S)$, and then returns $\left\{S^{*}, V \backslash S^{*}\right\}$ if $S^{*} \neq V$ and $\{V\}$ otherwise. Note that $S^{*} \in \operatorname{argmax}_{S \subseteq V} f_{1 / 2}(S)$ can be computed in polynomial time using our exact algorithms to Problem 1 . Then we can show that the algorithm gives a 1/3-approximate solution. In fact, the output of the algorithm is a $\frac{2}{3 k}$-approximate solution for any $k \geq 2$, as shown in Theorem 4 below.

In the case where $k \geq 3$, it is more practical to modify our algorithm so that we can find a partition of size more than 2 whose modularity density is better than that of the partition $\left\{S^{*}, V \backslash S^{*}\right\}$. One possible way is to iteratively find a subset with maximum $f_{1 / 2}$ in the remaining graph as long as the size constraint is satisfied and the modularity density increases. For reference, we describe the procedure in Algorithm 4.

Theorem 4. Algorithm 4 is a polynomial-time $\frac{2}{3 k}$-approximation algorithm for the $k$-modularity density maximization problem.

Proof. Let $S^{*} \in \operatorname{argmax}_{S \subseteq V} f_{1 / 2}(S)$. Let $C^{*}$ be an optimal solution to the $k$-modularity density maximization problem. Since the

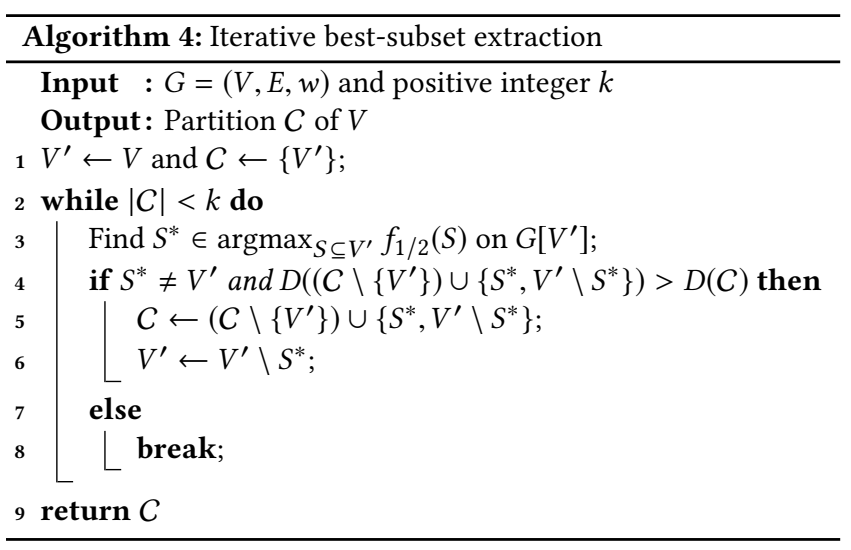

number of clusters contained in $C^{*}$ is at most $k$, we have

$$
D\left(C^{*}\right) \leq k \cdot 2 \cdot f_{1 / 2}\left(S^{*}\right) .
$$

Hence, if $S^{*}=V$ holds, then the output of our algorithm, i.e., $\{V\}$, is a $1 / k$-approximate solution. In what follows, we assume that $S^{*} \neq V$ holds. It suffices to show that $D\left(\left\{S^{*}, V \backslash S^{*}\right\}\right)=2 \cdot f_{1 / 2}\left(S^{*}\right)+2$. $f_{1 / 2}\left(V \backslash S^{*}\right) \geq \frac{2}{3 k} \cdot D\left(C^{*}\right)$.

Choose an arbitrary vertex $v \in V \backslash S^{*}$. Since $S^{*}$ maximizes $f_{1 / 2}(S)$, it holds that

$$
\frac{2 \cdot w_{\text {in }}\left[S^{*}\right]-w_{\text {cut }}\left[S^{*}\right]}{\left|S^{*}\right|} \geq \frac{2 \cdot w_{\text {in }}\left[S^{*} \cup\{v\}\right]-w_{\text {cut }}\left[S^{*} \cup\{v\}\right]}{\left|S^{*}\right|+1} .
$$

By using the facts that $w_{\text {in }}\left[S^{*} \cup\{v\}\right]=w_{\text {in }}\left[S^{*}\right]+d_{S^{*} \cup\{v\}}(v)$ and $w_{\text {cut }}\left[S^{*} \cup\{v\}\right]=w_{\text {cut }}\left[S^{*}\right]-d_{S^{*} \cup\{v\}}(v)+d_{V \backslash S^{*}}(v)$, the above inequality can be transformed into

$3 \cdot d_{S^{*} \cup\{v\}}(v)-d_{V \backslash S^{*}}(v) \leq \frac{2 \cdot w_{\text {in }}\left[S^{*}\right]-w_{\text {cut }}\left[S^{*}\right]}{\left|S^{*}\right|}=2 \cdot f_{1 / 2}\left(S^{*}\right)$.

Using this inequality, we have

$$
\begin{aligned}
2 \cdot f_{1 / 2}\left(V \backslash S^{*}\right) & =\frac{2 \cdot w_{\text {in }}\left[V \backslash S^{*}\right]-w_{\text {cut }}\left[V \backslash S^{*}\right]}{n-\left|S^{*}\right|} \\
& =\frac{2 \cdot w_{\text {in }}\left[V \backslash S^{*}\right]-\sum_{v \in V \backslash S^{*}} d_{S^{*} \cup\{v\}}(v)}{n-\left|S^{*}\right|} \\
& \geq \frac{2 \cdot w_{\text {in }}\left[V \backslash S^{*}\right]-\sum_{v \in V \backslash S^{*}} \frac{2 \cdot f_{1 / 2}\left(S^{*}\right)+d_{V \backslash S^{*}}(v)}{3}}{n-\left|S^{*}\right|} \\
& =-\frac{1}{3} \cdot 2 \cdot f_{1 / 2}\left(S^{*}\right)+\frac{4}{3} \cdot \frac{w_{\text {in }}\left[V \backslash S^{*}\right]}{n-\left|S^{*}\right|} \\
& \geq-\frac{1}{3} \cdot 2 \cdot f_{1 / 2}\left(S^{*}\right) .
\end{aligned}
$$

Therefore, we have

$$
\begin{aligned}
D\left(\left\{S^{*}, V \backslash S^{*}\right\}\right) & =2 \cdot f_{1 / 2}\left(S^{*}\right)+2 \cdot f_{1 / 2}\left(V \backslash S^{*}\right) \\
& \geq \frac{2}{3} \cdot 2 \cdot f_{1 / 2}\left(S^{*}\right) \geq \frac{2}{3 k} \cdot D\left(C^{*}\right),
\end{aligned}
$$

where the last inequality follows from inequality (4). This completes the proof.

\section{EXPERIMENTAL EVALUATION}

The purpose of our experiments is to verify the validity of our optimization model and to evaluate the performance of our greedy 
Table 2: Real-world graphs used in our experiments.

\begin{tabular}{lrrl}
\hline Name & $n$ & $m$ & Description \\
\hline Karate & 34 & 78 & Social network \\
Polbooks & 105 & 441 & Co-purchased \\
Adjnoun & 112 & 425 & Word adjacency \\
Football & 115 & 613 & Sports Games \\
Jazz & 198 & 2,742 & Social network \\
Email & 1,133 & 5,451 & Email \\
Polblogs & 1,222 & 16,714 & Blog \\
ego-Facebook & 4,039 & 88,234 & Social network \\
Wiki-Vote & 7,066 & 100,736 & Voting \\
ca-AstroPh & 17,903 & 196,972 & Collaboration \\
ca-CondMat & 21,363 & 91,286 & Collaboration \\
AS-22july06 & 22,963 & 48,436 & Internet topology \\
soc-Epinions1 & 75,877 & 405,739 & Social network \\
soc-Slashdot0902 & 82,168 & 504,230 & Social network \\
web-Stanford & 255,265 & $1,941,926$ & Web graph \\
com-DBLP & 317,080 & $1,049,866$ & Co-authorship \\
web-NotreDame & 325,729 & $1,090,108$ & Web graph \\
web-BerkStan & 654,782 & $6,581,871$ & Web graph \\
web-Google & 855,802 & $4,291,352$ & Web graph \\
AS-Skitter & $1,694,616$ & $11,094,209$ & Internet topology \\
com-Livejournal & $3,997,962$ & $34,681,189$ & Social network \\
soc-Livejournal & $4,843,953$ & $42,845,684$ & Social network \\
\hline
\end{tabular}

peeling algorithm (Algorithm 3 in Section 4). Throughout the experiments, we set $\alpha=0.2$ in our optimization model. In our preliminary experiments, we confirmed that the quality of the output of our algorithms is not sensitive to the choice of $\alpha$; indeed, the output is not drastically changed if we choose $\alpha$ from $[0.1,0.3]$. Table 2 lists graphs on which our experiments were conducted, most of which can be found in Leskovec and Krevl [31]. Note that all graphs here were made simple, unweighted, and undirected (if necessary) by ignoring the weight and the direction of edges and by removing self-loops and redundant multiple edges. Furthermore, if a graph is not connected, we take only the largest connected component in the graph.

\subsection{Validity of Optimization Model}

We first verify the validity of our optimization model. To this end, we compare optimal solutions of our model and the densest subgraph problem for graphs with at most 100,000 edges in Table 2. For both problems, optimal solutions are computed by the LP-based algorithms in Section 3 and by Charikar [9], respectively, in which LPs are solved using a state-of-the-art mathematical programming solver, Gurobi Optimizer 7.5.1, with the default parameter setting.

Table 3 lists the results of experiments. In the presentation of our experimental results, we use the average degree, double the density, rather than the density itself, to make it comparable with the average cut. As can be seen, for most graphs, the optimal solution to our optimization model has almost the same density but has smaller cut size (and smaller average cut), compared with those of the densest subgraph. The results present a desired trend to our purpose and support the validity of our optimization model. As will be demonstrated below, the trend becomes significant for relatively large graphs.

\subsection{Performance of Approximation Algorithm}

We next evaluate the performance of our greedy peeling algorithm. To this end, we compare solutions obtained by our algorithm and solutions obtained by the greedy peeling algorithm for the densest subgraph problem, which we refer to as DS-Greedy, on all graphs in Table 2.

Table 4 lists the results of experiments. For the results of our algorithm, the average degree is written in bold if it is greater than $80 \%$, compared with that of the corresponding solution obtained by DS-Greedy, and the (average) cut size is written in bold if it is less than $20 \%$. As can be seen, our algorithm has a strong tendency to extract a dense subgraph with small cut size (and small average cut). This trend is significant if a given graph is relatively large. For example, for the Web graphs, our algorithm finds a solution with almost the same density but much smaller cut size, compared with DS-Greedy. In particular, for web-NotreDame, our algorithm finds a subset of vertices with more than $96.1 \%$ density and less than $4.8 \%$ cut size, and for web-Google, it finds a subset of vertices with more than $99.1 \%$ density and less than $3.1 \%$ cut size. Finally, as for the quality of solutions, we see that both of our algorithm and DS-Greedy obtain almost optimal solutions. In fact, the objective function values on graphs with at most 100,000 edges almost attain the optimal values presented in Table 3 .

We here discuss the difference between greedy peeling strategies in DS-Greedy and our algorithm. Recall that DS-Greedy focuses only on the internal degree, while our algorithm takes into account the cut size in addition to it. To see the significance of this modification, in Figure 2, we plot the sequences of subsets produced by DS-Greedy and our algorithm for the Web graphs. We take the cut size and the average cut as the vertical axes, respectively, and the average degree as the horizontal axis. The objective function value $f_{\alpha}$ increases if we go to the lower-right direction; the blue and red bullets represent the outputs of DS-Greedy and our algorithm, respectively. As can be seen, our peeling strategy produces the sequence of subsets drastically different from the one by DS-Greedy. Indeed, our sequence contains a large number of dense subgraphs with small cut size (and small average cut) that do not appear in the sequence produced by the standard strategy. This observation indicates that changing a peeling strategy is essential to obtain a dense subgraph with sparse cut.

Finally, we report the scalability of our algorithm. Consistent with the running time analysis in Section 4, our algorithm is sufficiently scalable to large graphs with tens of millions of edges. Indeed, our algorithm outputs a solution within 10 minutes even for soc-LiveJournal, the largest graph with more than $42 \mathrm{M}$ edges. All experiments were conducted on a MacBook Pro with Intel Corei7 $2.8 \mathrm{GHz}$ CPU and 16 GB RAM. The code was written in Python.

\section{CONCLUSION}

In this study, we have proposed a new quality function for community detection in graphs, that is relevant to the modularity density for global community detection. Our quality function captures the 
Table 3: Difference of the optimal solutions.

\begin{tabular}{|c|c|c|c|c|c|c|c|c|c|}
\hline \multirow[b]{2}{*}{ Name } & \multicolumn{4}{|c|}{ Densest subgraph problem } & \multicolumn{5}{|c|}{ Our problem $(\alpha=0.2)$} \\
\hline & $\left|S^{*}\right|$ & $\frac{2 \cdot w_{\text {in }}\left[S^{*}\right]}{\left|S^{*}\right|}$ & $w_{\text {cut }}\left[S^{*}\right]$ & $\frac{w_{\text {cut }}\left[S^{*}\right]}{\left|S^{*}\right|}$ & $\left|S^{*}\right|$ & $\frac{\left.2 \cdot w_{\text {in }} \mid S^{*}\right]}{\left|S^{*}\right|}$ & $w_{\text {cut }}\left[S^{*}\right]$ & $\frac{\left.w_{\text {cut }} \mid S^{*}\right]}{\left|S^{*}\right|}$ & $f_{\alpha}\left(S^{*}\right)$ \\
\hline Karate & 16 & 5.25 & 29 & 1.81 & 28 & 4.79 & 5 & 0.18 & 2.36 \\
\hline Polbooks & 24 & 9.50 & 63 & 2.63 & 32 & 9.25 & 37 & 1.16 & 4.39 \\
\hline Adjnoun & 48 & 9.58 & 142 & 2.96 & 70 & 9.31 & 83 & 1.19 & 4.42 \\
\hline Football & 115 & 10.66 & 0 & 0.00 & 115 & 10.66 & 0 & 0.00 & 5.33 \\
\hline Jazz & 100 & 33.96 & 476 & 4.76 & 102 & 33.84 & 423 & 4.15 & 16.09 \\
\hline Email & 301 & 15.53 & 1,689 & 5.61 & 400 & 15.03 & 1,423 & 3.56 & 6.80 \\
\hline Polblogs & 139 & 55.97 & 3,577 & 25.73 & 393 & 53.84 & 5,200 & 13.23 & 24.27 \\
\hline ego-Facebook & 202 & 154.69 & 2,222 & 11.00 & 209 & 154.17 & 1,793 & 8.58 & 75.37 \\
\hline ca-CondMat & 30 & 26.73 & 331 & 11.03 & 35 & 26.06 & 308 & 8.80 & 11.27 \\
\hline AS-22july06 & 104 & 39.88 & 24,168 & 232.38 & 447 & 21.92 & 10,939 & 24.47 & 6.07 \\
\hline
\end{tabular}

Table 4: Difference of the approximate solutions.

\begin{tabular}{|c|c|c|c|c|c|c|c|c|c|}
\hline \multirow[b]{2}{*}{ Name } & \multicolumn{4}{|c|}{ Densest subgraph problem } & \multicolumn{5}{|c|}{ Our problem $(\alpha=0.2)$} \\
\hline & $|S|$ & $\frac{2 \cdot w_{\text {in }}[S]}{|S|}$ & $w_{\text {cut }}[S]$ & $\frac{\frac{w_{\text {cut }}[S]}{|S|}}{|S|}$ & $|S|$ & $\frac{2 \cdot w_{\text {in }}|S|}{|S|}$ & $w_{\text {cut }}[S]$ & $\frac{\frac{w_{\text {cut }}|S|}{|S|}}{|S|}$ & $f_{\alpha}(S)$ \\
\hline Karate & 18 & 5.22 & 25 & 1.39 & 18 & 5.22 & 25 & 1.39 & 2.33 \\
\hline Polbooks & 60 & 9.30 & 91 & 1.52 & 63 & 9.21 & 81 & 1.29 & 4.35 \\
\hline Adjnoun & 46 & 9.57 & 150 & 3.26 & 66 & 9.39 & 94 & 1.42 & 4.41 \\
\hline Football & 115 & 10.66 & 0 & 0.00 & 115 & 10.66 & 0 & 0.00 & 5.33 \\
\hline Jazz & 100 & 33.96 & 476 & 4.76 & 93 & 33.76 & 523 & 5.62 & 15.76 \\
\hline Email & 240 & 15.44 & 1,823 & 7.60 & 398 & 14.89 & 1,382 & 3.47 & 6.75 \\
\hline Polblogs & 279 & 55.82 & 6,918 & 24.80 & 402 & 53.23 & 4,907 & 12.21 & 24.18 \\
\hline ego-Facebook & 202 & 154.69 & 2,222 & 11.00 & 210 & 153.99 & 1,740 & 8.29 & 75.34 \\
\hline Wiki-Vote & 846 & 92.55 & 44,462 & 52.56 & 1,484 & 86.28 & 29,849 & 20.11 & 39.12 \\
\hline ca-AstroPh & 1,184 & 59.23 & 36,262 & 30.63 & 3,330 & 52.67 & 41,141 & 12.35 & 23.87 \\
\hline ca-CondMat & 26 & 25.00 & 385 & 14.81 & 46 & 22.17 & 306 & 6.65 & 9.76 \\
\hline AS-22july06 & 104 & 39.88 & 24,168 & 232.38 & 603 & 14.38 & 6,715 & 11.14 & 4.97 \\
\hline soc-Epinions 1 & 999 & 120.50 & 151,818 & 151.97 & 2,871 & 98.79 & 134,789 & 46.95 & 40.00 \\
\hline soc-Slashdot0902 & 219 & 86.89 & 42,417 & 193.68 & 6,181 & 64.52 & 213,471 & 34.54 & 25.35 \\
\hline web-Stanford & 547 & 118.37 & 30,748 & 56.21 & 830 & 113.57 & 18,757 & 22.60 & 52.26 \\
\hline com-DBLP & 114 & 113.00 & 2,073 & 18.18 & 111 & 110.00 & 2,048 & 18.45 & 51.31 \\
\hline web-NotreDame & 1,367 & 157.32 & 37,415 & 27.37 & 451 & 151.34 & 1,805 & 4.00 & 74.87 \\
\hline web-BerkStan & 392 & 206.81 & 236,523 & 603.38 & 4,592 & 167.16 & 46,922 & 10.22 & 81.53 \\
\hline web-Google & 240 & 54.36 & 10,524 & 43.85 & 61 & 53.90 & 316 & 5.18 & 25.91 \\
\hline AS-Skitter & 427 & 178.23 & 821,215 & $1,923.22$ & 203 & 90.34 & 1,030 & 5.07 & 44.16 \\
\hline com-Livejournal & 386 & 381.97 & 15,919 & 41.24 & 389 & 383.42 & 15,122 & 38.87 & 183.93 \\
\hline soc-LiveJournal & 503 & 454.95 & 58,455 & 116.21 & 779 & 388.96 & 57,742 & 74.12 & 179.66 \\
\hline
\end{tabular}

intuition of community structure using the density and the average cut. We then formulated an optimization model with the quality function. For the model, we have presented two exact algorithms: an LP-based algorithm and a flow-based algorithm. We have also designed an almost-linear-time approximation algorithm with theoretical performance guarantee by adopting a greedy peeling strategy. Furthermore, we have extended our algorithms to global community detection, that yields an approximation algorithm for the $k$-modularity density maximization problem. Computational experiments using well-known real-world graphs demonstrate that our algorithms are highly effective in obtaining a dense subgraph with sparse cut.

\section{ACKNOWLEDGMENTS}

The authors would like to thank the anonymous reviewers for their valuable comments. This work was supported by JST ERATO Grant Number JPMJER1201, Japan, and JSPS KAKENHI Grant Number JP18H05291. A.M. is supported by a Grant-in-Aid for Research Activity Start-up (No. 17H07357). N.K. is supported by a Grant-inAid for Scientific Research (C) (No. 17K00028).

\section{REFERENCES}

[1] R. Andersen and K. Chellapilla. 2009. Finding dense subgraphs with size bounds. In Proc. WAW'09. 25-37.

[2] A. Angel, N. Sarkas, N. Koudas, and D. Srivastava. 2012. Dense subgraph maintenance under streaming edge weight updates for real-time story identification. In 

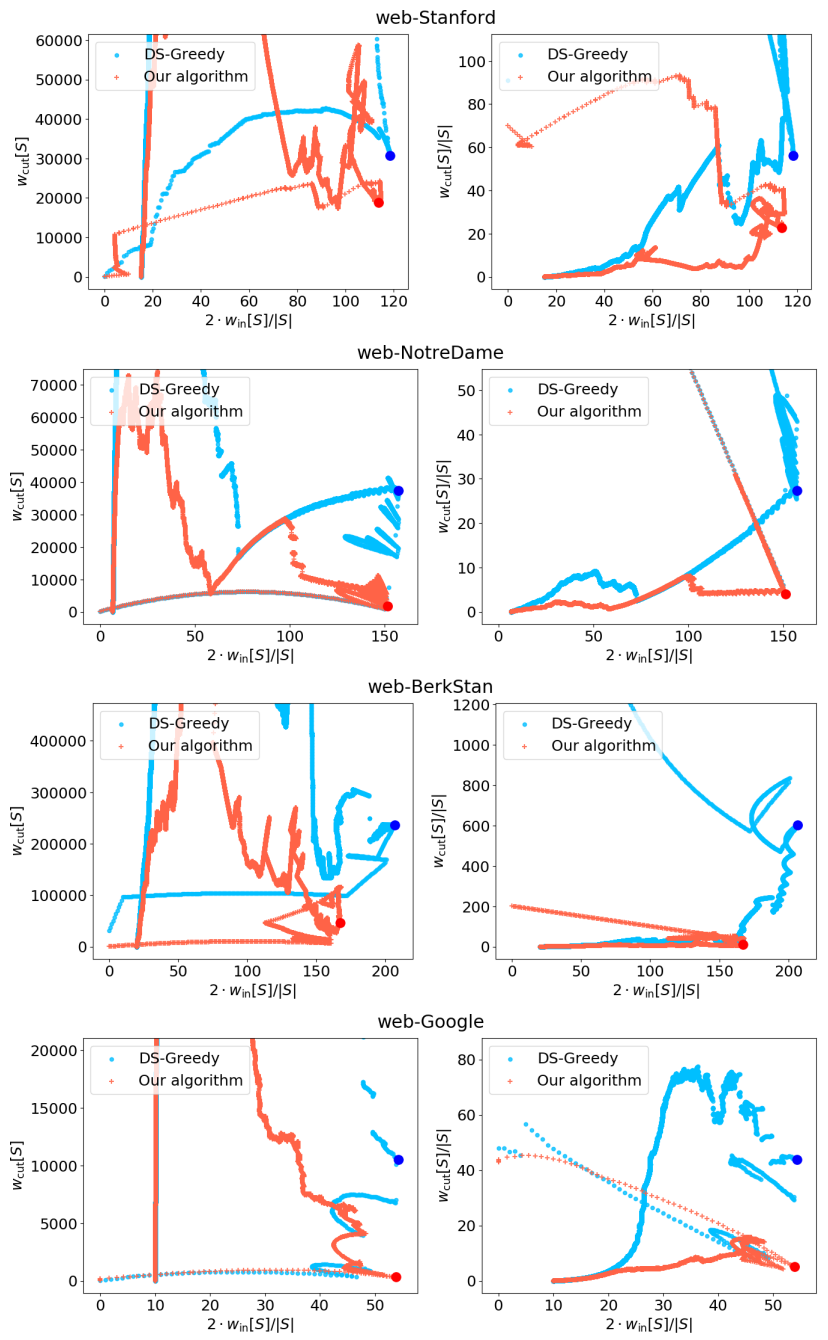

Figure 2: Difference of the greedy peeling strategies.

Proc. VLDB '12. 574-585.

[3] S. Arora, S. Rao, and U. Vazirani. 2009. Expander flows, geometric embeddings and graph partitioning. F. ACM 56, 2 (2009), 5:1-5:37.

[4] Y. Asahiro, K. Iwama, H. Tamaki, and T. Tokuyama. 2000. Greedily finding a dense subgraph. F. Algorithms 34, 2 (2000), 203-221.

[5] B. Bahmani, R. Kumar, and S. Vassilvitskii. 2012. Densest subgraph in streaming and MapReduce. In Proc. VLDB '12. 454-465.

[6] A. Bhaskara, M. Charikar, E. Chlamtac, U. Feige, and A. Vijayaraghavan. 2010 Detecting high log-densities: An $O\left(n^{1 / 4}\right)$ approximation for densest $k$-subgraph. In Proc. STOC '10. 201-210.

[7] S. Bhattacharya, M. Henzinger, D. Nanongkai, and C. E. Tsourakakis. 2015. Spaceand time-efficient algorithm for maintaining dense subgraphs on one-pass dynamic streams. In Proc. STOC '15. 173-182.

[8] T. Chakraborty, A. Dalmia, A. Mukherjee, and N. Ganguly. 2017. Metrics for community analysis: A survey. ACM Comput. Surv. 50, 4 (2017), 54:1-54:37.

[9] M. Charikar. 2000. Greedy approximation algorithms for finding dense components in a graph. In Proc. APPROX '00. 84-95.

[10] J. Cheriyan, T. Hagerup, and K. Mehlhorn. 1996. An $o\left(n^{3}\right)$-time maximum-flow algorithm. SIAM 7. Comput. 25, 6 (1996), 1144-1170.

[11] A. Costa. 2015. MILP formulations for the modularity density maximization problem. Eur. 7. Oper. Res. 245, 1 (2015), $14-21$.

[12] A. Costa, S. Kushnarev, L. Liberti, and Z. Sun. 2016. Divisive heuristic for modularity density maximization. Comput. Oper. Res. 71 (2016), 100-109.
[13] Y. Dourisboure, F. Geraci, and M. Pellegrini. 2007. Extraction and classification of dense communities in the web. In Proc. WWW'07. 461-470.

[14] A. Epasto, S. Lattanzi, and M. Sozio. 2015. Efficient densest subgraph computation in evolving graphs. In Proc. $W W W^{\prime}$ '15. 300-310.

[15] U. Feige, D. Peleg, and G. Kortsarz. 2001. The dense $k$-subgraph problem. Algorithmica 29, 3 (2001), 410-421.

[16] S. Fortunato. 2010. Community detection in graphs. Phys. Rep. 486, 3 (2010), $75-174$.

[17] S. Fortunato and D. Hric. 2016. Community detection in networks: A user guide. Phys. Rep. 659 (2016), 1-44.

[18] M. L. Fredman and R. E. Tarjan. 1987. Fibonacci heaps and their uses in improved network optimization algorithms. 7. ACM 34, 3 (1987), 596-615.

[19] S. Fujishige. 2005. Submodular Functions and Optimization. Annals of Discrete Mathematics, Vol. 58. Elsevier.

[20] E. Galimberti, F. Bonchi, and F. Gullo. 2017. Core decomposition and densest subgraph in multilayer networks. In Proc. CIKM '17. 1807-1816.

[21] M. R. Garey and D. S. Johnson. 1979. Computers and Intractability: A Guide to the Theory of NP-Completeness. W. H. Freeman \& Co., NY.

[22] D. Gibson, R. Kumar, and A. Tomkins. 2005. Discovering large dense subgraphs in massive graphs. In Proc. VLDB '05. 721-732.

[23] M. Girvan and M. E. J. Newman. 2002. Community structure in social and biological networks. Proc. Natl. Acad. Sci. U.S.A. 99, 12 (2002), 7821-7826.

[24] A. V. Goldberg. 1984. Finding a maximum density subgraph. University of California Berkeley.

[25] S. Hu, X. Wu, and T-H. H. Chan. 2017. Maintaining densest subsets efficiently in evolving hypergraphs. In Proc. CIKM '17. 929-938.

[26] D. J. H. Huang and A. B. Kahng. 1995. When clusters meet partitions: New density-based methods for circuit decomposition. In Proc. EDTC '95. 60-64.

[27] S. Iwata. 2008. Submodular function minimization. Math. Program. 112, 1 (2008), 45-64.

[28] Y. Kawase and A. Miyauchi. 2017. The densest subgraph problem with a convex/concave size function. Algorithmica (2017). https://doi.org/10.1007/ s00453-017-0400-7

[29] S. Khuller and B. Saha. 2009. On finding dense subgraphs. In Proc. ICALP '09. 597-608.

[30] Y. T. Lee, A. Sidford, and S. C. W. Wong. 2015. A faster cutting plane method and its implications for combinatorial and convex optimization. In Proc. FOCS '15. 1049-1065.

[31] J. Leskovec and A. Krevl. 2014. SNAP Datasets: Stanford Large Network Dataset Collection. http://snap.stanford.edu/data.

[32] J. Leskovec, K. J. Lang, A. Dasgupta, and M. W. Mahoney. 2008. Statistical properties of community structure in large social and information networks. In Proc. WWW'08. 695-704.

[33] J. Leskovec, K. J. Lang, and M. W. Mahoney. 2010. Empirical comparison of algorithms for network community detection. In Proc. WWW'10. 631-640.

[34] Z. Li, S. Zhang, R.-S. Wang, X.-S. Zhang, and L. Chen. 2008. Quantitative function for community detection. Phys. Rev. E 77 (2008), 036109.

[35] Z. Li, S. Zhang, R.-S. Wang, X.-S. Zhang, and L. Chen. 2015. Erratum: Quantitative function for community detection [Phys. Rev. E 77, 036109 (2008)]. Phys. Rev. E 91 (2015), 019901.

[36] A. McGregor, D. Tench, S. Vorotnikova, and H. T. Vu. 2015. Densest subgraph in dynamic graph streams. In Proc. MFCS '15. 472-482.

[37] M. Mitzenmacher, J. Pachocki, R. Peng, C. E. Tsourakakis, and S. C. Xu. 2015. Scalable large near-clique detection in large-scale networks via sampling. In Proc. KDD '15. 815-824.

[38] A. Miyauchi, Y. Iwamasa, T. Fukunaga, and N. Kakimura. 2015. Threshold influence model for allocating advertising budgets. In Proc. ICML '15. 1395-1404.

[39] A. Miyauchi and Y. Kawase. 2015. What is a network community? A novel quality function and detection algorithms. In Proc. CIKM '15. 1471-1480.

[40] M. A. U. Nasir, A. Gionis, G. De Francisci Morales, and S. Girdzijauskas. 2017. Fully dynamic algorithm for top-k densest subgraphs. In Proc. CIKM '17. 1817-1826.

[41] M. E. J. Newman and M. Girvan. 2004. Finding and evaluating community structure in networks. Phys. Rev. E 69 (2004), 026113.

[42] R. Santiago and L. C. Lamb. 2017. Efficient modularity density heuristics for large graphs. Eur. F. Oper. Res. 258, 3 (2017), 844-865.

[43] R. Santiago and L. C. Lamb. 2017. Exact computational solution of modularity density maximization by effective column generation. Comput. Oper. Res. 86 (2017), 18-29.

[44] J. Šíma and S. E. Schaeffer. 2006. On the NP-completeness of some graph cluster measures. In Proc. SOFSEM '06. 530-537.

[45] C. E. Tsourakakis. 2015. The k-clique densest subgraph problem. In Proc. WWW'15. 1122-1132.

[46] C. E. Tsourakakis, F. Bonchi, A. Gionis, F. Gullo, and M. Tsiarli. 2013. Denser than the densest subgraph: Extracting optimal quasi-cliques with quality guarantees. In Proc. KDD '13. 104-112.

[47] Z. Zou. 2013. Polynomial-time algorithm for finding densest subgraphs in uncertain graphs. In Proc. MLG '13. No page numbers. 